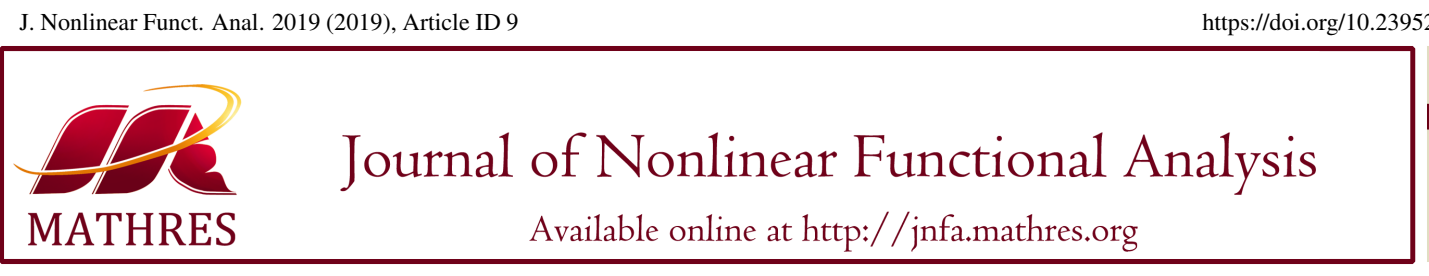

https://doi.org/10.23952/jnfa.2019.9

\title{
LINEAR CONVERGENCE OF AN ITERATIVE ALGORITHM FOR SOLVING THE MULTIPLE-SETS SPLIT EQUALITY PROBLEM
}

\author{
MEILING FENG, LUOYI SHI*, RUDONG CHEN
}

Department of Mathematics, Tianjin Polytechnic University, Tianjin 300387, China

\begin{abstract}
In this paper, we propose a subgradient projection algorithm for solving the multiple-sets split equality problem (MSSEP), and investigate its linear convergence. We involve the bounded linear regularity for the MSSEP, and construct several sufficient conditions to ensure the linear convergence of our proposed algorithm. One of the highlights of our algorithm is that metric projections onto given feasibility sets are easily calculated (that is, the projections onto half-spaces). Some numerical results are provided to illustrate the validity of our proposed algorithm.
\end{abstract}

Keywords. Linear convergence; Bounded linear regularity; Multiple-sets split equality problem; Subgradient projection algorithm.

2010 Mathematics Subject Classification. 47H05, 90C33.

\section{INTRODUCTION}

Let $H_{1}, H_{2}$ and $H_{3}$ be three real Hilbert spaces. Let $C$ and $Q$ be nonempty closed and convex subsets of $H_{1}$ and $H_{2}$, respectively. Let $A: H_{1} \rightarrow H_{3}$ and $B: H_{2} \rightarrow H_{3}$ be bounded and linear operators. The split equality problem (in short, SEP), as a generalization of the split feasibility problem, was proposed by Moudafi [1], which is formulated as

$$
\text { finding } x \in C \text { and } y \in Q \text { such that } A x=B y \text {. }
$$

This class of problem has received much attention due to its broad applications, such as, intensitymodulated radiation therapy, decomposition methods for partial differential equations, and applications in game theory, etc.

Recently, various algorithms were introduced to solve the split equality problem. In 2013, Byrne and Moudafi [2] proposed the following algorithms:

\footnotetext{
* Corresponding author.

E-mail addresses: 979868374@qq.com (M. Feng), shiluoyi@tjpu.edu.cn (L. Shi), chenrd@tjpu.edu.cn (R. Chen).

Received September 22, 2018; Accepted February 22, 2019.
} 
simultaneous CQ-algorithm:

$$
\left\{\begin{array}{l}
x_{k+1}=P_{C}\left(x_{k}-\gamma_{k} A^{*}\left(A x_{k}-B y_{k}\right)\right) \\
y_{k+1}=P_{Q}\left(y_{k}+\gamma_{k} B^{*}\left(A x_{k}-B y_{k}\right)\right)
\end{array}\right.
$$

relaxed simultaneous CQ-algorithm:

$$
\left\{\begin{array}{l}
x_{k+1}=P_{C_{k}}\left(x_{k}-\gamma_{k} A^{*}\left(A x_{k}-B y_{k}\right)\right), \\
y_{k+1}=P_{Q_{k}}\left(y_{k}+\gamma_{k} B^{*}\left(A x_{k}-B y_{k}\right)\right),
\end{array}\right.
$$

where $P_{C}\left(P_{C_{k}}\right)$ and $P_{Q}\left(P_{Q_{k}}\right)$ are the projection from $H_{1}$ onto $C\left(C_{k}\right)$ and the projection from $H_{2}$ onto $Q$ $\left(Q_{k}\right)$, respectively, $A^{*}$ and $B^{*}$ are the adjoint operators of $A$ and $B$, respectively. They proved that above algorithms converge weakly to a solution of SEP (1.1). In 2014, in order to obtain the strong convergence of the algorithm for the SEP, Shi [3] proposed the following algorithm:

$$
\left\{\begin{array}{l}
x_{k+1}=P_{C}\left[\left(1-\alpha_{k}\right)\left(x_{k}-\gamma_{k} A^{*}\left(A x_{k}-B y_{k}\right)\right)\right], \\
y_{k+1}=P_{Q}\left[\left(1-\alpha_{k}\right)\left(y_{k}+\gamma_{k} B^{*}\left(A x_{k}-B y_{k}\right)\right)\right] .
\end{array}\right.
$$

Recently, much attention has been focused on this field, we refer the reader to $[4,5,6,7,8,9,10,11$, 12] and the references therein. However, few results involved the rate of convergence. In this paper, we investigate the multiple-sets split equality problem (MSSEP), which can be characterized mathematically as following

$$
\text { finding } x \in \bigcap_{i=1}^{t} C_{i} \text { and } y \in \bigcap_{j=1}^{r} Q_{j} \text { such that } A x=B y,
$$

where $r$ and $t$ are positive integers, $\left\{C_{i}\right\}_{i=1}^{t}$ and $\left\{Q_{j}\right\}_{j=1}^{r}$ are nonempty closed and convex subsets of Hilbert spaces $H_{1}$ and $H_{2}$, respectively, and $H_{3}$ is also a Hilbert space, both operators $A: H_{1} \rightarrow H_{3}$ and $B: H_{2} \rightarrow H_{3}$ are bounded and linear. Obviously, when $t=r=1$, the MSSEP reduces to the SEP (1.1). If $t=r=1$ and $B=I$, then the MSSEP is reduced to the SFP, which was introduced by Censor and Elfving [13]. Without loss of generality, we may assume $t>r$, and choose $Q_{r+1}=Q_{r+2}=\cdots=Q_{t}=H_{2}$. Let $S_{i}=C_{i} \times Q_{i} \subseteq H=H_{1} \times H_{2}, i=1,2, \cdots, t, S=\bigcap_{i=1}^{t} S_{i}, G=[A,-B]: H \rightarrow H_{3}, G^{*}$ be the adjoint operator of $G$. Then problem (1.2) can be reformulated as

$$
\text { finding } w=(x, y) \in S \text { which satisfies } G w=0 .
$$

Tian [14] proposed the following split self-adaptive step size algorithm to solve it:

$$
w_{k+1}=w_{k}+\frac{\rho_{1, k} \sum_{i=1}^{t} \alpha_{i}\left\|P_{S_{i}} w_{k}-w_{k}\right\|^{2}}{\left\|\sum_{i=1}^{t} \alpha_{i}\left(P_{S_{i}} w_{k}-w_{k}\right)\right\|^{2}} \sum_{i=1}^{t} \alpha_{i}\left(P_{S_{i}} w_{k}-w_{k}\right)-\frac{\rho_{2, k}\left\|G w_{k}\right\|^{2}}{\left\|G^{*} G w_{k}\right\|^{2}} G^{*} G w_{k},
$$

where $0<\underline{\rho}_{1} \leq \rho_{1, k} \leq \bar{\rho}_{1}<1,0<\underline{\rho}_{2} \leq \rho_{2, k} \leq \bar{\rho}_{2}<1,\left\{\alpha_{i}\right\}_{i=1}^{t}>0$. We remark here that the algorithm only converges weakly to a solution of MSSEP (1.3). And the rate of convergence was not investigated. In addition, the orthogonal projections $P_{C_{i}}(i=1,2, \cdots, t)$ and $P_{Q_{j}}(j=1,2, \cdots, t)$ are usually difficult to be calculated. Based on the above disadvantages, we propose a subgradient projection algorithm for solving the MSSEP (1.3) by using projections onto half-spaces to replace the original convex sets, which are very practical, and we also consider the linear convergence of the algorithm proposed.

The general structure of the paper is as follows. In Section 2, we involve the concept of bounded linear regularity for the MSSEP (1.3) and present some relevant definitions and lemmas which will be needed 
for our convergence analysis. In Section 3, we propose a subgradient projection algorithm and give its linear convergence. In Section 4, some numerical results are presented to illustrate the validity of our proposed algorithm.

\section{PRELIMINARIES}

Let $H$ be a real Hilbert space with inner product $\langle\cdot, \cdot\rangle$ and norm $\|\cdot\|$, respectively. $I$ denotes the identity operator on $H$. For a set $S \subset H$, int $S$ denotes the interior of $S$. We denote by $\mathbf{B}$ and $\overline{\mathbf{B}}$ to the unit open metric ball and unit closed metric ball with center at origin, respectively, that is,

$$
\mathbf{B}:=\{v \in H:\|v\|<1\} \text { and } \overline{\mathbf{B}}:=\{v \in H:\|v\| \leq 1\} .
$$

For an element $w \in H$ and a set $S \subset H$, the classical metric projection of $w$ onto $S$ and the distance from $w$ onto $S$, denoted by $P_{S}(w)$ and $d_{S}(w)$, respectively, which are defined by

$$
P_{S}(w):=\arg \min \{\|w-v\|: v \in S\} \text { and } d_{S}(w):=\inf \{\|w-v\|: v \in S\} .
$$

The following lemma presents several important properties of the projection operator.

Lemma 2.1. [15] Let $C$ be a closed, convex, and nonempty subset of $H$. Then, for any $x, y \in H$ and $z \in C$,

(i) $\left\langle x-P_{C} x, z-P_{C} x\right\rangle \leq 0$;

(ii) $\left\|P_{C} x-P_{C} y\right\|^{2} \leq\left\langle P_{C} x-P_{C} y, x-y\right\rangle$;

(iii) $\left\|P_{C} x-z\right\|^{2} \leq\|x-z\|^{2}-\left\|P_{C} x-x\right\|^{2}$.

Let $G: H \rightarrow H_{3}$ be a bounded linear operator. The kernel of $G$ is denoted by $\operatorname{ker} G=\{x \in H: G x=0\}$, and the orthogonal complement of $\operatorname{ker} G$ is denoted by $(\operatorname{ker} G)^{\perp}=\{y \in H:\langle x, y\rangle=0$ for any $x \in \operatorname{ker} G\}$. It is known that both $\operatorname{ker} G$ and $(\operatorname{ker} G)^{\perp}$ are closed subspaces of $H$.

The aim of this section is to construct several sufficient conditions to ensure the linear convergence of the subgradient projection algorithm for MSSEP (1.3). Throughout this section, we use $\Gamma$ to denote the solution set of MSSEP (1.3), that is,

$$
\Gamma:=S \cap G^{-1}(0)=\{w \in S: G w=0\},
$$

and assume that the MSSEP is consistent. Thus, $\Gamma$ is a nonempty closed and convex set.

Recall that a sequence $\left\{w_{k}\right\}$ in $H$ is said to be converge linearly to its limit $w$ (with rate $\beta \in[0,1)$ ) if there exists $\alpha>0$ and a positive integer $N$ such that

$$
\left\|w_{k}-w\right\| \leq \alpha \beta^{k} \quad \text { for all } k \geq N .
$$

Next, we will give the concept of bounded linear regularity.

Definition 2.2. [16] Let $\left\{S_{i}\right\}_{i \in I}$ be a family of closed convex subsets of a real Hilbert space $H$ and $S=\bigcap_{i \in I} S_{i} \neq \emptyset$. The family $\left\{S_{i}\right\}_{i \in I}$ is said to be boundedly linearly regular if, for each $r>0$, there exists a constant $\gamma_{r}>0$ such that

$$
d_{S}(w) \leq \gamma_{r} \sup \left\{d_{S_{i}}(w): i \in I\right\} \quad \text { for all } w \in r \mathbf{B} .
$$

Lemma 2.3. [17] Let $\left\{S_{i}\right\}_{i \in I}$ be a family of closed convex subsets of a real Hilbert space $H$. If $S_{i} \cap$ $\operatorname{int}\left(\bigcap_{j \in I \backslash\{i\}} S_{j}\right) \neq \emptyset$, then the family $\left\{S_{i}\right\}_{i \in I}$ is boundedly linearly regular. $j \in I \backslash\{i\}$ 
Definition 2.4. The MSSEP is said to satisfy bounded linear regularity property if, for each $r>0$, there exists a constant $\tau_{r}>0$ such that

$$
\tau_{r} d_{\Gamma}(w) \leq \max \left\{d_{S}(w),\|G w\|\right\} \quad \text { for all } w \in r \mathbf{B} .
$$

Lemma 2.5. [18] Let $G: H \rightarrow H_{3}$ be a bounded linear operator. Then $G$ is injective and has closed range if and only if $G$ is bounded below, i.e., there exists a positive constant $\gamma$ such that $\|G w\| \geq \gamma\|w\|$ for all $w \in H$.

Lemma 2.6. Let $\{S, \operatorname{ker} G\}$ be boundedly linearly regular and $G$ has closed range. Then the MSSEP (1.3) satisfies the bounded linear regularity property.

Proof. Since $\{S, \operatorname{ker} G\}$ is boundedly linearly regular, for any $r>0$, there exists $\tau_{r}>0$ such that

$$
d_{\Gamma}(w)=d_{S \cap \operatorname{ker} G}(w) \leq \tau_{r} \max \left\{d_{S}(w), d_{\operatorname{ker} G}(w)\right\} \quad \text { for all } w \in r B .
$$

Since $G$ restricted to $(\operatorname{ker} G)^{\perp}$ is injective and has closed range, it follows from Lemma 2.5 that there exists $v>0$ such that

$$
\left\|G w_{1}\right\| \geq v\left\|w_{1}\right\| \quad \text { for all } w_{1} \in(\operatorname{ker} G)^{\perp}
$$

Hence,

$$
d_{G^{-1}(0)}(w) \leq \frac{1}{v}\|G w\| \quad \text { for all } w \in H .
$$

Combining (2.2) and (2.3), we obtain

$$
d_{\Gamma}(w) \leq \tau_{r} \max \left\{d_{S}(w), \frac{1}{v}\|G w\|\right\} \quad \text { for all } w \in r B .
$$

Next, the proof is divided into two cases.

Case1: suppose that $\frac{1}{v}<1$. Then

$$
d_{\Gamma}(w) \leq \tau_{r} \max \left\{d_{S}(w), \frac{1}{v}\|G w\|\right\} \leq \tau_{r} \max \left\{d_{S}(w),\|G w\|\right\} \quad \text { for all } w \in r B .
$$

That is,

$$
\frac{1}{\tau_{r}} d_{\Gamma}(w) \leq \max \left\{d_{S}(w),\|G w\|\right\} \quad \text { for all } w \in r B .
$$

Case2: suppose that $\frac{1}{v} \geq 1$. Then

$$
d_{\Gamma}(w) \leq \tau_{r} \max \left\{d_{S}(w), \frac{1}{v}\|G w\|\right\} \leq \frac{\tau_{r}}{v} \max \left\{d_{S}(w),\|G w\|\right\} \quad \text { for all } w \in r B .
$$

That is,

$$
\frac{v}{\tau_{r}} d_{\Gamma}(w) \leq \max \left\{d_{S}(w),\|G w\|\right\} \quad \text { for all } w \in r B
$$

The proof is complete.

Now, we are in a position to present the definition of subdifferential which is vital for constructing linear convergence later.

Definition 2.7. [17] Let $f: H \rightarrow R$ be a convex function. The subdifferential of $f$ at $x$ is defined as

$$
\partial f(x):=\{\xi \in H: f(y) \geq f(x)+\langle\xi, y-x\rangle \text { for all } y \in H\} .
$$


Lemma 2.8. [17] Let $f: H \rightarrow R$ be a convex function, $x_{0} \in H$, and $f$ be subdifferentiable at $x_{0}$. Suppose that $C=\{x \in H: f(x) \leq 0\}$ is nonempty for any $g\left(x_{0}\right) \in \partial f\left(x_{0}\right)$, and define $S$ by

$$
S:=C\left(f, x_{0}, g\left(x_{0}\right)\right):=\left\{x \in H: f\left(x_{0}\right)+\left\langle g\left(x_{0}\right), x-x_{0}\right\rangle \leq 0\right\} .
$$

Then,

(i) $C \subseteq S$. If $g\left(x_{0}\right) \neq 0$, then $S$ is a halfspace; otherwise, $S=H$;

(ii) $P_{S}\left(x_{0}\right)=x_{0}-\frac{\max \left\{f\left(x_{0}\right), 0\right\}}{\left\|g\left(x_{0}\right)\right\|^{2}} g\left(x_{0}\right)$;

(iii) $d_{S}\left(x_{0}\right)=\frac{\max \left\{f\left(x_{0}\right), 0\right\}}{\left\|g\left(x_{0}\right)\right\|}$.

The following equality and concept of Fejér monotone sequence is essential.

Lemma 2.9. [15] Let $\left\{x_{n}\right\}_{n \in I}$ be a finite family in $H$, and $\left\{\lambda_{n}\right\}_{n \in I}$ be a finite family in $R$ with $\sum_{n \in I} \lambda_{n}=1$.

Then the following equality holds:

$$
\left\|\sum_{n \in I} \lambda_{n} x_{n}\right\|^{2}=\sum_{n \in I} \lambda_{n}\left\|x_{n}\right\|^{2}-\frac{1}{2} \sum_{n \in I} \sum_{m \in I} \lambda_{n} \lambda_{m}\left\|x_{n}-x_{m}\right\|^{2}, n \geq 2 .
$$

Definition 2.10. [15] Let $C$ be a nonempty subset of $H$, and $\left\{x_{k}\right\}$ be a sequence in $H$. $\left\{x_{k}\right\}$ is said to be Fejér monotone with respect to $C$, if

$$
\left\|x_{k+1}-z\right\| \leq\left\|x_{k}-z\right\|, \forall z \in C .
$$

Obviously, a Fejér monotone sequence $\left\{x_{k}\right\}$ is bounded and $\lim _{k \rightarrow \infty}\left\|x_{k}-z\right\|$ exists.

\section{MAIN RESUlts}

In this section, we will propose the subgradient projection algorithm and show that the algorithm converges linearly to a solution of MSSEP (1.3). Without loss of generality, the sets $C_{i}$ and $Q_{j}$ could be expressed as

$$
C_{i}:=\left\{x \in H_{1}: c_{i}(x) \leq 0\right\}
$$

and

$$
Q_{j}:=\left\{y \in H_{2}: q_{j}(y) \leq 0\right\}
$$

where $c_{i}: H_{1} \rightarrow R$ and $q_{j}: H_{2} \rightarrow R$ are convex functions, for all $i, j=1,2, \cdots, t$ ( $t$ is positive integer). Suppose that both $c_{i}$ and $q_{j}$ are subdifferentiable on $H_{1}$ and $H_{2}$, respectively, and that $\partial c_{i}$ and $\partial q_{j}$ are bounded operators (i.e., bounded on bounded sets). Define:

$$
C_{i, k}:=\left\{x \in H_{1}: c_{i}\left(x_{k}\right)+\left\langle\xi_{i, k}, x-x_{k}\right\rangle \leq 0\right\},
$$

where $\xi_{i, k} \in \partial c_{i}\left(x_{k}\right), i=1,2, \cdots, t$, and

$$
Q_{j, k}:=\left\{y \in H_{2}: q_{j}\left(y_{k}\right)+\left\langle\eta_{j, k}, y-y_{k}\right\rangle \leq 0\right\},
$$

where $\eta_{j, k} \in \partial q_{j}\left(y_{k}\right), j=1,2, \cdots, t$.

Obviously, $C_{i} \subseteq C_{i, k}$ and $Q_{j} \subseteq Q_{j, k}$ for all $k \in \mathbf{N}$ and $i, j=1,2, \cdots, t$. Notice that $C_{i, k}$ and $Q_{j, k}$ are half-spaces and therefore the corresponding metric projections have closed forms. Since $C_{i, k}$ and $Q_{j, k}$ have specific forms, the metric projections onto $C_{i, k}$ and $Q_{j, k}$ can be calculated directly (see the Lemma 2.8).

Let $S_{i}=C_{i} \times Q_{i}$ and $S_{i, k}=C_{i, k} \times Q_{i, k}$ for all $k \in \mathbf{N}$ and $i=1,2, \cdots, t$. Then, we have $S_{i} \subseteq S_{i, k}$, and $S_{i, k}$ is half-space for all $k \in \mathbf{N}$ and $i=1,2, \cdots, t$; 
Dang [19] defined the proximity function $p(x, y)$ of the MSSEP (1.2) as follows:

$$
p(x, y):=\frac{1}{2} \sum_{i=1}^{t} \alpha_{i}\left\|P_{C_{i}} x-x\right\|^{2}+\frac{1}{2} \sum_{j=1}^{r} \lambda_{j}\left\|P_{Q_{j}} y-y\right\|^{2}+\frac{1}{2}\|A x-B y\|^{2},
$$

where $\alpha_{i}>0$ for all $i=1,2, \cdots, t$ and $\lambda_{j}>0$ for all $j=1,2, \cdots, r$ with $\sum_{i=1}^{t} \alpha_{i}+\sum_{j=1}^{r} \lambda_{j}=1 . C_{i}$ and $Q_{j}$ are defined by (3.1) and (3.2), respectively. Hence, the function $p(x, y)$ is convex and differentiable with gradient

$$
\nabla p(x, y)=\left(\sum_{i=1}^{t} \alpha_{i}\left(x-P_{C_{i}} x\right)+A^{*}(A x-B y), \sum_{j=1}^{r} \lambda_{j}\left(y-P_{Q_{j}} y\right)-B^{*}(A x-B y)\right)^{\mathrm{T}} .
$$

They constructed following iterative algorithm for the MSSEP (1.2):

$$
\left\{\begin{array}{l}
x_{k+1}=P_{\Omega_{1}}\left[x_{k}-\gamma\left(\sum_{i=1}^{t} \alpha_{i}\left(x_{k}-P_{C_{i}, k} x_{k}\right)+A^{*}\left(A x_{k}-B y_{k}\right)\right)\right], \\
y_{k+1}=P_{\Omega_{2}}\left[y_{k}-\gamma\left(\sum_{i=1}^{r} \lambda_{i}\left(y_{k}-P_{Q_{i}, k} y_{k}\right)-B^{*}\left(A x_{k+1}-B y_{k}\right)\right)\right],
\end{array}\right.
$$

where

$$
\gamma \in\left(0, \min \left\{\frac{1}{4\|A\|^{2}}, \frac{1}{2}, \frac{1}{4\|B\|^{2}}\right\}\right),
$$

$\Omega_{1} \subset H_{1}$ and $\Omega_{2} \subset H_{2}$ are auxiliary simple sets.

Now, we use the modification of (3.3) to give our subgradient projection algorithm for the MSSEP (1.3).

Algorithm 3.1. For an arbitrarily initial point $w_{0}=\left(x_{0}, y_{0}\right) \in H$, the sequence $\left\{w_{k+1}\right\}$ is generated by

$$
w_{k+1}=w_{k}-\gamma_{k}\left(\sum_{i=1}^{t} \alpha_{i}\left(w_{k}-P_{S_{i}, k} w_{k}\right)+G^{*} G w_{k}\right),
$$

or component-wise

$$
\left\{\begin{array}{l}
x_{k+1}=x_{k}-\gamma_{k}\left(\sum_{i=1}^{t} \alpha_{i}\left(x_{k}-P_{C_{i}, k} x_{k}\right)+A^{*}\left(A x_{k}-B y_{k}\right)\right), \\
y_{k+1}=y_{k}-\gamma_{k}\left(\sum_{i=1}^{t} \alpha_{i}\left(y_{k}-P_{Q_{i}, k} y_{k}\right)-B^{*}\left(A x_{k}-B y_{k}\right)\right),
\end{array}\right.
$$

where, at each iteration $k$ :

(i) $0<\lim _{k \rightarrow \infty} \inf \gamma_{k} \leq \lim _{k \rightarrow \infty} \sup \gamma_{k}<\min \left\{1, \frac{1}{\|G\|^{2}}\right\}$;

(ii) $\left\{\alpha_{i}\right\}_{i=1}^{t} \subset(0,+\infty)$ and $\sum_{i=1}^{t} \alpha_{i}=1$.

Theorem 3.2. Let the MSSEP (1.3) satisfy the bounded linear regularity property, and the sequence $\left\{w_{k}\right\}$ be defined by Algorithm 3.1. If the following conditions are satisfied:

(a) $\left\{w_{k}\right\}$ is linearly focusing, that is, there exists $\beta>0$ such that

$$
\beta d_{S_{i}}\left(w_{k}\right) \leq d_{S_{i, k}}\left(w_{k}\right) \quad \text { for any } i \in\{1,2, \cdots, t\} ;
$$

(b) There is $w=(x, y) \in S_{i}$, such that $c_{r}(x)<0, q_{r}(y)<0, r \in\{1,2, \cdots, t\} \backslash\{i\}$, i.e., $S_{i} \bigcap \operatorname{int}\left(\bigcap_{r \in I \backslash\{i\}} S_{r}\right) \neq$ $\emptyset(I=\{1,2, \cdots, t\})$.

Then, $\left\{w_{k}\right\}$ converges linearly to a solution of MSSEP (1.3). 
Proof. First, we will show that the sequence $\left\{w_{k}\right\}$ is Fejér monotone with respect to $\Gamma$.

Taking $\bar{w} \in \Gamma$, one has $G \bar{w}=0$, and

$$
\begin{aligned}
& \left\|w_{k+1}-\bar{w}\right\|^{2} \\
& =\left\|w_{k}-\gamma_{k}\left(\sum_{i=1}^{t} \alpha_{i}\left(w_{k}-P_{S_{i}, k} w_{k}\right)+G^{*} G w_{k}\right)-\bar{w}\right\|^{2} \\
& =\left\|w_{k}-\bar{w}\right\|^{2}-2 \gamma_{k}\left\langle w_{k}-\bar{w}, \sum_{i=1}^{t} \alpha_{i}\left(w_{k}-P_{S_{i}, k} w_{k}\right)+G^{*} G w_{k}\right\rangle \\
& \quad+\gamma_{k}^{2}\left\|\sum_{i=1}^{t} \alpha_{i}\left(w_{k}-P_{S_{i}, k} w_{k}\right)+G^{*} G w_{k}\right\|^{2} \\
& \leq\left\|w_{k}-\bar{w}\right\|^{2}+2 \gamma_{k}^{2}\left\|\sum_{i=1}^{t} \alpha_{i}\left(w_{k}-P_{S_{i}, k} w_{k}\right)\right\|^{2}+2 \gamma_{k}^{2}\left\|G^{*} G w_{k}\right\|^{2} \\
& \quad-2 \gamma_{k}\left\langle w_{k}-\bar{w}, \sum_{i=1}^{t} \alpha_{i}\left(w_{k}-P_{S_{i}, k} w_{k}\right)\right\rangle-2 \gamma_{k}\left\langle w_{k}-\bar{w}, G^{*} G w_{k}\right\rangle .
\end{aligned}
$$

By Lemma 2.9, we have

$$
\begin{aligned}
& \left\|\sum_{i=1}^{t} \alpha_{i}\left(w_{k}-P_{S_{i}, k} w_{k}\right)\right\|^{2} \\
& =\sum_{i=1}^{t} \alpha_{i}\left\|w_{k}-P_{S_{i}, k} w_{k}\right\|^{2}-\frac{1}{2} \sum_{i=1}^{t} \sum_{j=1}^{t} \alpha_{i} \alpha_{j}\left\|\left(w_{k}-P_{S_{i}, k} w_{k}\right)-\left(w_{k}-P_{S_{j}, k} w_{k}\right)\right\|^{2} \\
& \leq \sum_{i=1}^{t} \alpha_{i}\left\|w_{k}-P_{S_{i}, k} w_{k}\right\|^{2} .
\end{aligned}
$$

Hence,

$$
\begin{aligned}
\left\|w_{k+1}-\bar{w}\right\|^{2} \leq & \left\|w_{k}-\bar{w}\right\|^{2}+2 \gamma_{k}^{2} \sum_{i=1}^{t} \alpha_{i}\left\|w_{k}-P_{S_{i, k}} w_{k}\right\|^{2}+2 \gamma_{k}^{2}\left\|G^{*} G w_{k}\right\|^{2} \\
& -2 \gamma_{k}\left\langle w_{k}-\bar{w}, \sum_{i=1}^{t} \alpha_{i}\left(w_{k}-P_{S_{i, k}} w_{k}\right)\right\rangle-2 \gamma_{k}\left\langle w_{k}-\bar{w}, G^{*} G w_{k}\right\rangle .
\end{aligned}
$$

From the properties of the projection operator (i.e., Lemma 2.1) and the definition of the adjoint operator, we get the following estimations:

$$
\begin{aligned}
\left\langle w_{k}-\bar{w}, \sum_{i=1}^{t} \alpha_{i}\left(w_{k}-P_{S_{i}, k} w_{k}\right)\right\rangle & =\sum_{i=1}^{t} \alpha_{i}\left\langle w_{k}-\bar{w}, w_{k}-P_{S_{i}, k} w_{k}\right\rangle \\
& =\sum_{i=1}^{t} \alpha_{i}\left(\left\langle w_{k}-P_{S_{i}, k} w_{k}, w_{k}-P_{S_{i}, k} w_{k}\right\rangle+\left\langle P_{S_{i}, k} w_{k}-\bar{w}, w_{k}-P_{S_{i}, k} w_{k}\right\rangle\right) \\
& =\sum_{i=1}^{t} \alpha_{i}\left(\left\|w_{k}-P_{S_{i}, k} w_{k}\right\|^{2}+\left\langle P_{S_{i}, k} w_{k}-\bar{w}, w_{k}-P_{S_{i}, k} w_{k}\right\rangle\right) \\
& \geq \sum_{i=1}^{t} \alpha_{i}\left\|w_{k}-P_{S_{i}, k} w_{k}\right\|^{2}
\end{aligned}
$$

and

$$
\left\langle w_{k}-\bar{w}, G^{*} G w_{k}\right\rangle=\left\langle G w_{k}-G \bar{w}, G w_{k}\right\rangle=\left\|G w_{k}\right\|^{2}
$$


Substituting (3.6) and (3.7) into (3.5), we obtain

$$
\begin{aligned}
\left\|w_{k+1}-\bar{w}\right\|^{2} \leq & \left\|w_{k}-\bar{w}\right\|^{2}+2 \gamma_{k}^{2} \sum_{i=1}^{t} \alpha_{i}\left\|w_{k}-P_{S_{i}, k} w_{k}\right\|^{2}+2 \gamma_{k}^{2}\left\|G^{*} G w_{k}\right\|^{2} \\
& -2 \gamma_{k} \sum_{i=1}^{t} \alpha_{i}\left\|w_{k}-P_{S_{i}, k} w_{k}\right\|^{2}-2 \gamma_{k}\left\|G w_{k}\right\|^{2} \\
= & \left\|w_{k}-\bar{w}\right\|^{2}-2 \gamma_{k}\left(1-\gamma_{k}\right) \sum_{i=1}^{t} \alpha_{i}\left\|w_{k}-P_{S_{i}, k} w_{k}\right\|^{2}-2 \gamma_{k}\left(1-\gamma_{k} \frac{\left\|G^{*} G w_{k}\right\|^{2}}{\left\|G w_{k}\right\|^{2}}\right)\left\|G w_{k}\right\|^{2} .
\end{aligned}
$$

According to (i) in Algorithm 3.1, we conclude from (3.8) that

$$
\left\|w_{k+1}-\bar{w}\right\| \leq\left\|w_{k}-\bar{w}\right\| \text {. }
$$

Hence, the sequence $\left\{w_{k}\right\}$ is Fejér monotone with respect to $\Gamma$. It follows that $\left\{w_{k}\right\}$ is bounded and $\lim _{k \rightarrow \infty}\left\|w_{k}-\bar{w}\right\|$ exists.

Next, we show that $\left\{w_{k}\right\}$ converges linearly to a solution of MSSEP (1.3).

Since $\bar{w}$ is taken arbitrarily in $\Gamma$, we obtain from (3.8) that

$$
d_{\Gamma}^{2}\left(w_{k+1}\right) \leq d_{\Gamma}^{2}\left(w_{k}\right)-2 \gamma_{k}\left(1-\gamma_{k}\right) \sum_{i=1}^{t} \alpha_{i} d_{S_{i}, k}^{2}\left(w_{k}\right)-2 \gamma_{k}\left(1-\gamma_{k} \frac{\left\|G^{*} G w_{k}\right\|^{2}}{\left\|G w_{k}\right\|^{2}}\right)\left\|G w_{k}\right\|^{2} .
$$

Note that $\left\{w_{k}\right\}$ is linearly focusing, there exists $\beta>0$ such that

$$
\beta d_{S_{i}}\left(w_{k}\right) \leq d_{S_{i, k}}\left(w_{k}\right) \quad \text { for all } i \in\{1,2, \cdots, t\} .
$$

We see from condition (b) that $S_{i} \bigcap \operatorname{int}\left(\bigcap_{r \in I \backslash\{i\}} S_{r}\right) \neq \emptyset$. By Lemma 2.3, we obtain that $\left\{S_{i}\right\}_{i=1}^{t}$ is boundedly linearly regular. By Definition 2.2, there exists $\tau>0$ such that

$$
d_{S}\left(w_{k}\right) \leq \tau \max \left\{d_{S_{i}}\left(w_{k}\right), i=1,2, \cdots, t\right\},
$$

that is,

$$
\frac{1}{\tau} d_{S}\left(w_{k}\right) \leq \max \left\{d_{S_{i}}\left(w_{k}\right), i=1,2, \cdots, t\right\} .
$$

Substituting (3.10) and (3.11) into (3.9), we obtain

$$
\begin{aligned}
d_{\Gamma}^{2}\left(w_{k+1}\right) & \leq d_{\Gamma}^{2}\left(w_{k}\right)-2 \gamma_{k}\left(1-\gamma_{k}\right) \sum_{i=1}^{t} \alpha_{i} \beta^{2} d_{S_{i}}^{2}\left(w_{k}\right)-2 \gamma_{k}\left(1-\gamma_{k} \frac{\left\|G^{*} G w_{k}\right\|^{2}}{\left\|G w_{k}\right\|^{2}}\right)\left\|G w_{k}\right\|^{2} \\
& \leq d_{\Gamma}^{2}\left(w_{k}\right)-2 \gamma_{k}\left(1-\gamma_{k}\right) \alpha \beta^{2} \max \left\{d_{S_{i}}^{2}\left(w_{k}\right), i \in I\right\}-2 \gamma_{k}\left(1-\gamma_{k} \frac{\left\|G^{*} G w_{k}\right\|^{2}}{\left\|G w_{k}\right\|^{2}}\right)\left\|G w_{k}\right\|^{2} \\
& \leq d_{\Gamma}^{2}\left(w_{k}\right)-2 \gamma_{k}\left(1-\gamma_{k}\right) \frac{\alpha \beta^{2}}{\tau^{2}} d_{S}^{2}\left(w_{k}\right)-2 \gamma_{k}\left(1-\gamma_{k} \frac{\left\|G^{*} G w_{k}\right\|^{2}}{\left\|G w_{k}\right\|^{2}}\right)\left\|G w_{k}\right\|^{2},
\end{aligned}
$$

where $\alpha=\min \left\{\alpha_{i}, i=1,2, \cdots, t\right\}$ and $I=\{1,2, \cdots, t\}$. From (i) in Algorithm 3.1, one deduces that

$$
\lim _{k \rightarrow \infty} \inf \left[\left(1-\gamma_{k}\right) \frac{\alpha \beta^{2}}{\tau^{2}}\right]>0,
$$

and

$$
\lim _{k \rightarrow \infty} \inf \left(1-\gamma_{k} \frac{\left\|G^{*} G w_{k}\right\|^{2}}{\left\|G w_{k}\right\|^{2}}\right)>0 .
$$

Thus, there exist $N$ and $M$ such that

$$
a_{1}:=\inf _{k \geq N}\left[\left(1-\gamma_{k}\right) \frac{\alpha \beta^{2}}{\tau^{2}}\right]>0,
$$


and

$$
a_{2}:=\inf _{k \geq M}\left(1-\gamma_{k} \frac{\left\|G^{*} G w_{k}\right\|^{2}}{\left\|G w_{k}\right\|^{2}}\right)>0 .
$$

Let $a:=\min \left\{a_{1}, a_{2}\right\}$, and $L:=\max \{N, M\}$, then inequality (3.12) is reduced to

$$
d_{\Gamma}^{2}\left(w_{k+1}\right) \leq d_{\Gamma}^{2}\left(w_{k}\right)-2 \gamma_{k} a\left(d_{S}^{2}\left(w_{k}\right)+\left\|G w_{k}\right\|^{2}\right) \quad \text { for all } k \geq L .
$$

Since the MSSEP satisfies bounded linear regularity property, there exists $v>0$ such that

$$
v d_{\Gamma}\left(w_{k}\right) \leq \max \left\{d_{S}\left(w_{k}\right),\left\|G w_{k}\right\|\right\} .
$$

It follows that

$$
v^{2} d_{\Gamma}^{2}\left(w_{k}\right) \leq d_{S}^{2}\left(w_{k}\right)+\left\|G w_{k}\right\|^{2} .
$$

Substituting (3.14) into (3.13), we get

$$
d_{\Gamma}^{2}\left(w_{k+1}\right) \leq d_{\Gamma}^{2}\left(w_{k}\right)-2 \gamma_{k} a v^{2} d_{\Gamma}^{2}\left(w_{k}\right)=\left(1-2 \gamma_{k} a v^{2}\right) d_{\Gamma}^{2}\left(w_{k}\right) \quad \text { for all } k \geq L .
$$

Let $b:=a v^{2}$. Then

$$
d_{\Gamma}^{2}\left(w_{k+1}\right) \leq\left(1-2 b \gamma_{k}\right) d_{\Gamma}^{2}\left(w_{k}\right) \leq d_{\Gamma}^{2}\left(w_{L}\right) \prod_{i=L+1}^{k}\left(1-2 b \gamma_{i}\right) \quad \text { for all } k \geq L .
$$

Obviously, for each $\bar{w} \in \Gamma,\left\|w_{k+1}-\bar{w}\right\|$ is monotone decreasing for $k$. Hence

$$
\begin{aligned}
\left\|w_{l}-w_{k}\right\| & \leq\left\|w_{l}-P_{\Gamma}\left(w_{k}\right)\right\|+\left\|w_{k}-P_{\Gamma}\left(w_{k}\right)\right\| \\
& \leq 2\left\|w_{k}-P_{\Gamma}\left(w_{k}\right)\right\|=2 d_{\Gamma}\left(w_{k}\right) \quad \text { for all } l>k .
\end{aligned}
$$

It follows that

$$
\left\|w_{l}-w_{k+1}\right\| \leq 2 d_{\Gamma}\left(w_{L}\right) \prod_{i=L+1}^{k} \sqrt{1-2 b \gamma_{i}} \quad \text { for all } l \geq k+1 .
$$

Let $q:=e^{-b} \in(0,1)$. Then

$$
\prod_{i=L+1}^{k} \sqrt{1-2 b \gamma_{i}}=\exp \left\{\frac{1}{2} \sum_{i=L+1}^{k} \ln \left(1-2 b \gamma_{i}\right)\right\} \leq q^{\sum_{i=L+1}^{k} \gamma_{i}} .
$$

Therefore,

$$
\left\|w_{l}-w_{k+1}\right\| \leq 2 d_{\Gamma}\left(w_{L}\right) q^{\sum_{i=L+1}^{k} \gamma_{i}} \quad \text { for all } l \geq k+1 .
$$

Since $0<\lim _{k \rightarrow \infty} \inf \gamma_{k} \leq \lim _{k \rightarrow \infty} \sup \gamma_{k}<\min \left\{1, \frac{1}{\|G\|^{2}}\right\}$, it follows that $\left\{w_{k}\right\}$ is a Cauchy sequence and converges to a solution $w^{*}$ of MSSEP (1.3) satisfying

$$
\left\|w_{k+1}-w^{*}\right\| \leq d_{\Gamma}\left(w_{L}\right) q^{\sum_{i=L+1}^{k} \gamma_{i}} \quad \text { for all } k \geq L .
$$

Let

$$
\delta:=\max \left\{d_{\Gamma}\left(w_{L}\right) q^{-\sum_{i=1}^{L} \gamma_{i}}, \max \left\{\left\|w_{i}-w^{*}\right\| q^{-\sum_{j=1}^{i} \gamma_{j}}, i=1,2, \ldots, L\right\}\right\}
$$

Then,

$$
\left\|w_{k}-w^{*}\right\| \leq \delta q^{\sum_{i=1}^{k} \gamma_{i}}
$$

Hence, $\left\{w_{k}\right\}$ converges linearly to $w^{*}$. The proof is complete.

If $t=1$ in Algorithm 3.1, we have the iterative algorithm for solving the SEP (1.1). 
Definition 3.3. The SEP is said to satisfy bounded linear regularity property if, for each $r>0$, there exists a constant $\tau_{r}>0$ such that

$$
\tau_{r} d_{\Gamma}(w) \leq \max \left\{d_{S}(w),\|G w\|\right\} \quad \text { for all } w \in r \mathbf{B} .
$$

where $S=C \times Q, G=[A,-B]$, and $w=(x, y) \in C \times Q$.

Corollary 3.4. Let SEP (1.1) satisfy the bounded linear regularity property (i.e., (3.15) holds). For an arbitrarily initial point $w_{0}=\left(x_{0}, y_{0}\right) \in H$, the sequence $\left\{w_{k}\right\}$ is defined by

$$
w_{k+1}=w_{k}-\gamma_{k}\left(\left(w_{k}-P_{S_{k}} w_{k}\right)+G^{*} G w_{k}\right),
$$

or component-wise

$$
\left\{\begin{array}{l}
x_{k+1}=x_{k}-\gamma_{k}\left(\left(x_{k}-P_{C_{k}} x_{k}\right)+A^{*}\left(A x_{k}-B y_{k}\right)\right), \\
y_{k+1}=y_{k}-\gamma_{k}\left(\left(y_{k}-P_{Q_{k}} y_{k}\right)-B^{*}\left(A x_{k}-B y_{k}\right)\right),
\end{array}\right.
$$

where $0<\lim _{k \rightarrow \infty} \inf \gamma_{k} \leq \lim _{k \rightarrow \infty} \sup \gamma_{k}<\min \left\{1, \frac{1}{\|G\|^{2}}\right\}$. Then, $\left\{w_{k}\right\}$ converges linearly to a solution of SEP (1.1).

\section{NUMERICAL EXPERIMENTS}

Let $H_{1}=\mathbb{R}, H_{2}=\mathbb{R}^{2}$ and $H_{3}=\mathbb{R}^{3}, c: H_{1} \rightarrow \mathbb{R}$ and $q: H_{2} \rightarrow \mathbb{R}$ are defined by

$$
c(x)=-x^{2} \text { and } q(y)=-\left(y_{1}^{2}+y_{2}^{2}\right) \quad \text { for all } x \in H_{1}, y=\left(y_{1}, y_{2}\right) \in H_{2} .
$$

Then $C=\{x \in \mathbb{R}: c(x) \leq 0\}=\mathbb{R}, Q=\left\{y \in \mathbb{R}^{2}: q(y) \leq 0\right\}=\mathbb{R}^{2}$. Note that $C \subseteq C_{k}$ and $Q \subseteq Q_{k}, C_{k}=\mathbb{R}$, $Q_{k}=\mathbb{R}^{2} . A: H_{1} \rightarrow H_{3}$ and $B: H_{2} \rightarrow H_{3}$ are defined by

$$
A(x)=(x, 0,0) \text { and } B(y, z)=(y, z, 0) \text { for all }(x, y, z) \in \mathbb{R}^{3},
$$

respectively. Let $S=C \times Q=\mathbb{R}^{3}, G=[A,-B]: H \rightarrow H_{3}$ be defined by

$$
G(x, y, z)=(x-y,-z, 0) \text { for all }(x, y, z) \in \mathbb{R}^{3} .
$$

Then $\operatorname{ker} G=\{(x, x, 0): x \in \mathbb{R}\} \neq \emptyset$, the range of $G$ is closed, and the solution set of SEP is

$$
\Gamma=(C \times Q) \bigcap \operatorname{ker} G=\{(x, x, 0): x \in \mathbb{R}\} .
$$

It is easy to check that the SEP satisfies the bounded linear regularity property.

Let $w_{0}=\left(x_{0}, y_{0}, z_{0}\right) \in C \times Q$. In view of equation (3.16), we have

$$
\begin{aligned}
& x_{k+1}=\left(1-\gamma_{k}\right) x_{k}+\gamma_{k} y_{k}, \\
& y_{k+1}=\left(1-\gamma_{k}\right) y_{k}+\gamma_{k} x_{k}, \\
& z_{k+1}=\left(1-\gamma_{k}\right) z_{k} .
\end{aligned}
$$

In algorithm (3.16), we take $\gamma_{k}=0.4, \frac{k}{k+1}$, respectively. Then we have the following numerical results (the $x$-coordinate denotes the number of iterations, and the $y$-coordinate denotes the logarithm of the error). The whole codes were written in Wolfram Mathematica (version 9.0). All the numerical results were performed on a personal computer with Intel(R) Core(TM)2 CPU 1.66GHz and RAM 2.00GB.

We choose error to be $10^{-5}, 10^{-10}$, and initial value $w_{0}=(4,8,3), w_{0}=(150,550,60)$, respectively. 


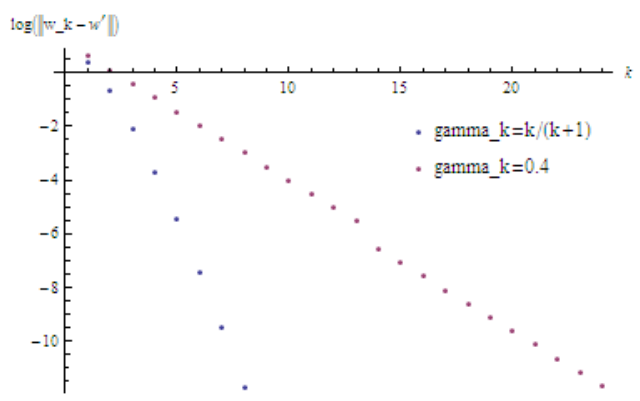

FIGURE 1. Error $=10^{-5}, x_{1}=4, y_{1}=8, z_{1}=3, w^{\prime}=(6,6,0)$

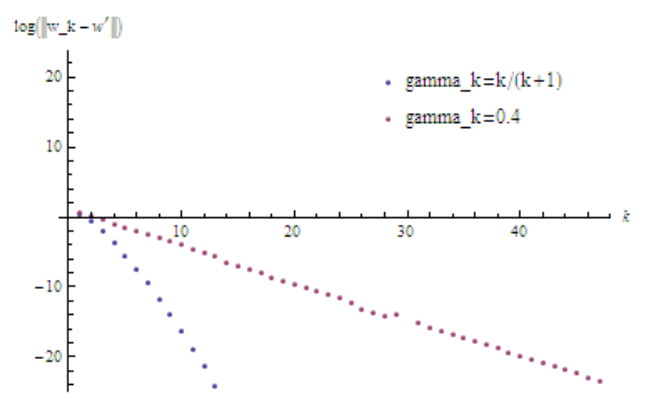

FIGURE 2. Error $=10^{-10}, x_{1}=4, y_{1}=8, z_{1}=3, w^{\prime}=(6,6,0)$

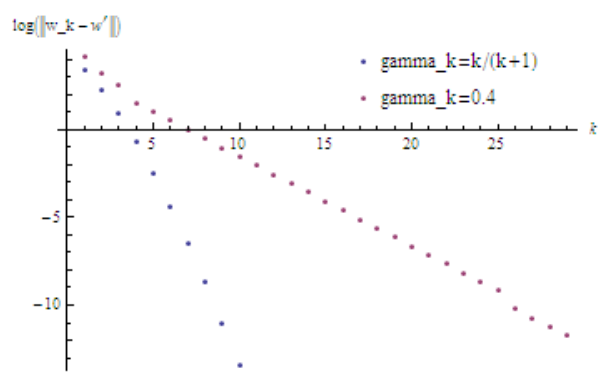

FIGURE 3. Error $=10^{-5}, x_{1}=150, y_{1}=550, z_{1}=60, w^{\prime}=(350,350,0)$

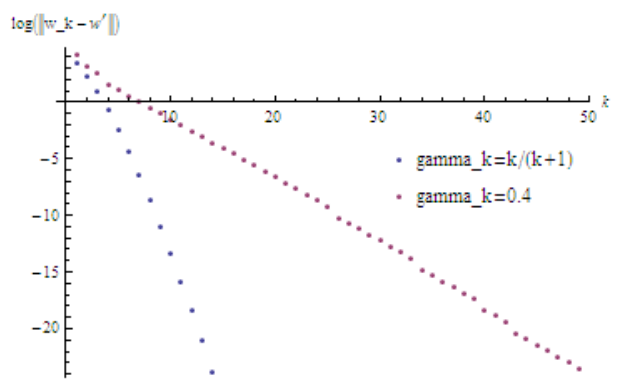

FIGURE 4. Error $=10^{-10}, x_{1}=150, y_{1}=550, z_{1}=60, w^{\prime}=(350,350,0)$ 


\section{Acknowledgement}

This paper was supported by NSFC Grants No.11301379, No.11226125 and No.11671167.

\section{REFERENCES}

[1] A. Moudafi, Alternating CQ algorithm for convex feasibility and split fixed point problems, J. Nonlineaer Convex Anal. 15 (2013), 809-818

[2] C. Byrne, A. Moudafi, Extensions of the CQ algorithm for split feasibility and split equality problems, Working paper UAG, 2013.

[3] L. Shi, R. Chen, Y.. Wu, Strong convergence of iterative algorithms for split equality problem, J. Inequal. Appl. 2014 (2014), Article ID 478.

[4] Q.L. Dong, S. He, J. Zhao, Solving the split equality problem without prior knowledge of operator norms, Optimization 64 (2015), 1887-1906

[5] Q.L. Dong, S. He, Modified projection algorithms for solving the split equality problems, Sci. World J. 2014 (2014), Article ID 328787.

[6] X. Zheng, Y. Yao, Y.C. Liou, L. Leng. Fixed point algorithms for the split problem of demicontractive operators, J. Nonlineaer Sci. Appl. 10 (2017), 1263-1269.

[7] Y. Yao, Y.C. Liou, J.C. Yao. Split common fixed point problem for two quasipseudo-contractive operators and its algorithm construction, Fixed Point Theory Appl. 2015 (2015), Article ID 127.

[8] Y. Yao, L. Leng, Y.C. Liou. Strong convergence of an iteration for the split common fixed points of demicontractive operators, J. Nonlinear Convex Anal. 19 (2018), 197-205.

[9] Y. Yao, R. Chen, G. Marino, Y.C. Liou. Applications of fixed point and optimization methods to the multiple-sets split feasibility problem, J. Appl. Math. 2012 (2012), 1-36.

[10] X. Qin, A. Petrusel, J.C. Yao, CQ iterative algorithms for fixed points of nonexpansive mappings and split feasibility problems in Hilbert spaces, J. Nonlinear Convex Anal. 19 (2018), 157-165.

[11] Y. Dang, Y. Gao, The strong convergence of a KM-CQ-like algorithm for split feasibility problem, Inverse Probl. 27 (2011), Article ID 015007.

[12] J. Zhao, Solving split equality fixed-point problem of quasi-nonexpansive mappings without prior knowledge of operators norms, Optimization, 64 (2015), 2619-2630

[13] Y. Censor, T. Elfving, A multiprojection algorithm using Bregman projections in a product space, Numer. Algorithms 8 (1994), 221-239

[14] D. Tian, L. Shi, R. Chen, Iterative algorithm for solving the multiple-sets split equality problem with split self-adaptive step size in Hilbert spaces, J. Inequal. Appl. 2016 (2016), Article ID 34.

[15] H.H. Bauschke, P.L. Combettes, Convex Analysis and Monotone Operator Theory in Hilbert Spaces, Springer, London, (2011)

[16] X. Zhao, K. Ng, C. Li, J.C. Yao, Linear regularity and linear convergence of projection-based methods for solving convex feasibility problems, Appl. Math. Optim. 78 (2018), 613-641.

[17] H.H. Bauschke, J.M. Borwein, On projection algorithms for solving convex feasibility problems. SIAM Rev. 38 (1996), 367-426

[18] J.B. Conway, A Course in Functional Analysis, Second edition, GTM 96, Springer, New York, (1989)

[19] Y.Z. Dang, J. Yao, Y. Gao, Relaxed two points projection method for solving the multiple-sets split equality problem, Numer. Algorithms 78 (2018), 263-275. 